\title{
Convolutional neural networks in medical image understanding: a survey
}

\author{
D. R. Sarvamangala ${ }^{1}\left(\mathbb{D} \cdot\right.$ Raghavendra V. Kulkarni $^{2}$
}

Received: 25 March 2020 / Revised: 5 October 2020 / Accepted: 22 November 2020 / Published online: 3 January 2021

(c) The Author(s), under exclusive licence to Springer-Verlag GmbH, DE part of Springer Nature 2021

\begin{abstract}
Imaging techniques are used to capture anomalies of the human body. The captured images must be understood for diagnosis, prognosis and treatment planning of the anomalies. Medical image understanding is generally performed by skilled medical professionals. However, the scarce availability of human experts and the fatigue and rough estimate procedures involved with them limit the effectiveness of image understanding performed by skilled medical professionals. Convolutional neural networks (CNNs) are effective tools for image understanding. They have outperformed human experts in many image understanding tasks. This article aims to provide a comprehensive survey of applications of CNNs in medical image understanding. The underlying objective is to motivate medical image understanding researchers to extensively apply CNNs in their research and diagnosis. A brief introduction to CNNs has been presented. A discussion on CNN and its various award-winning frameworks have been presented. The major medical image understanding tasks, namely image classification, segmentation, localization and detection have been introduced. Applications of CNN in medical image understanding of the ailments of brain, breast, lung and other organs have been surveyed critically and comprehensively. A critical discussion on some of the challenges is also presented.
\end{abstract}

Keywords Classification $\cdot$ Convolutional neural networks $\cdot$ Detection $\cdot$ Image understanding $\cdot$ Localization $\cdot$ Segmentation

\section{Introduction}

Loss of human lives can be prevented or the medical trauma experienced in an injury or a disease can be reduced through the timely diagnosis of medical anomalies. Medical anomalies include glaucoma, diabetic retinopathy, tumors [34], interstitial lung diseases [44], heart diseases and tuberculosis. Diagnosis and prognosis involve the understanding of the images of the affected area obtained using X-ray, magnetic resonance imaging (MRI), computed tomography (CT), positron emission tomography (PET), single photon emission computed tomography or ultrasound scanning. Image understanding involves the detection of anomalies, ascertaining their locations and borders, and estimating their

D. R. Sarvamangala

sarvamangala.dr@reva.edu.in

Raghavendra V. Kulkarni

arvie@ieee.org

1 REVA University, Bengaluru, India

2 Ramaiah University of Applied Sciences, Bengaluru, India sizes and severity. The scarce availability of human experts and their fatigue, high consultation charges and rough estimate procedures limit the effectiveness of image understanding. Further, shapes, locations and structures of the medical anomalies are highly variable [55]. This makes diagnosis difficult even for specialized physicians [4]. Therefore, human experts often feel a need for support tools to aid in precise understanding of medical images. This is the motivation for intelligent image understanding systems.

Image understanding systems that exploit machine learning (ML) techniques are fast evolving in recent years. ML techniques include decision tree learning [35], clustering, support vector machines (SVMs) [47], k-means nearest neighbor (K-NN), restricted Boltzmann machines (RBMs) [42] and random forests (RFs) [28]. The pre-requisite for ML techniques to work efficiently is the extraction of discriminant features. And these features are generally unknown and is also a very challenging task especially for applications involving image understanding and is still a topic of research. A logical step to overcome was to create intelligent machines which could learn features needed for image understanding and extract it on its own. One such intelligent 
and successful model is the convolutional neural network (CNN) model, which automatically learns the needed features and extracts it for medical image understanding. The CNN model is made of convolutional filters whose primary function is to learn and extract necessary features for efficient medical image understanding. CNN started gaining popularity in the year 2012, due to AlexNet [41], a CNN model, which defeated all the others models with a record accuracy and low error rate in imageNet challenge 2012. $\mathrm{CNN}$ has been used by corporate giants for providing internet services, automatic tagging in images, product recommendations, home feed personalization and autonomous cars [59]. The major applications of the $\mathrm{CNN}$ are in image and signal processing, natural language processing and data analytics. The CNN had a major breakthrough when GoogleNet used it to detect cancer at an accuracy of $89 \%$ while human pathologists could achieve the accuracy of only $70 \%$ [3].

\subsection{Motivation and purpose}

CNNs have contributed significantly in the areas of image understanding. CNN-based approaches are placed in the leader board of the many image understanding challenges, such as Medical Image Computing and Computer Assisted Intervention (MICCAI) biomedical challenge, Brain Tumor segmentation (BRATS) Multimodal Brain Tumor Segmentation challenge [48], Imagenet classification challenge, challenges of International Conference on Pattern Recognition (ICPR) [31] and Ischemic Stroke Lesion Segmentation (ISLES) challenge [32]. CNN has become a powerful choice as a technique for medical image understanding. Researchers have successfully applied CNNs for many medical image understanding applications like detection of tumors and their classification into benign and malignant [52], detection of skin lesions [50], detection of optical coherence tomography images [39], detection of colon cancer [71], blood cancer, anomalies of the heart [40], breast [36], chest, eye etc. Also CNN-based models like CheXNet [56, 58], used for classifying 14 different ailments of the chest achieved better results compared to the average performance of human experts.

CNNs have also dominated the area of COVID-19 detection using chest X-rays/CT scans. Research involving CNNs is now a dominant topic at major conferences. In addition, there are special issues reserved in reputed journals for solving challenges using deep learning models. The vast amount of literature available on CNNs is the testimonial of their efficiency and the widespread use. However, various research communities are developing these applications concurrently and the dissemination results are scattered in a wide and diverse range of conference proceedings and journals.

A large number of surveys on deep learning have been published recently. A review of deep learning techniques applied in medical imaging, bioinformatics and pervasive sensing has been presented in [60]. A thorough review of deep learning techniques for segmentation of MRI images of brain has been presented in [2]. Survey of deep learning techniques for medical image segmentation, their achievements and challenges involved in medical image segmentation has been presented in [27] Though literature is replete with many survey papers, most of them concentrate on deep learning models which include $\mathrm{CNN}$, recurrent neural network, generative adversial network or on a particular application. There is also no coverage of the application of CNN in early detection of COVID-19 as well as many other areas.

The survey includes research papers on various applications of CNNs in medical image understanding. The papers for the survey are queried from various journal websites. Additionally, arxiv, conference proceedings of various medical image challenges are also included in the survey. Also the references of these papers are checked. The query used are: "CNN" or "deep learning" or "convolutional neural network" or terms related to medical image understanding. These terms had to be present either in title or abstract to be considered.

The objective of this survey is to offer a comprehensive overview of applications and methodology of CNNs and its variants, in the fields of medical image understanding including the detection of latest global pandemic COVID19. The survey includes overview tables which can be used for quick reference. The authors leverage experiences of their own and that of the research fraternity on the applications of CNNs to provide an insight into various state of the art CNN models, challenges involved in designing CNN model, overview of research trends in the field, and to motivate medical image understanding researchers and medical professionals to extensively apply CNNs in their research and diagnosis, respectively.

\subsection{Contributions and the structure}

Primary contributions of this article are as follows:

1. To briefly introduce medical image understanding and CNN.

2. To convey that CNN has percolated in the field of medical image understanding.

3. To identify the various challenges in medical image understanding.

4. To highlight contributions of $\mathrm{CNN}$ to overcome those challenges

The remainder of this article has been organized as follows: Medical image understanding has been briefly introduced in Sect. 2. A brief introduction of CNN and its architecture has been presented in Sect. 3. The applications of CNN in 
medical image understanding have been surveyed comprehensively through Sects. 4-7. Finally, concluding remarks and a projection of the trends in CNN applications in image understanding have been presented in Sect. 8 .

\section{Medical image understanding}

Medical imaging is necessary for the visualization of internal organs for the detection of abnormalities in their anatomy or functioning. Medical image capturing devices, such as X-ray, CT, MRI, PET and ultrasound scanners capture the anatomy or functioning of the internal organs and present them as images or videos. The images and videos must be understood for the accurate detection of anomalies or the diagnosis of functional abnormalities. If an abnormality is detected, then its exact location, size and shape must be determined. These tasks are traditionally performed by the trained physicians based on their judgment and experience. Intelligent healthcare systems aim to perform these tasks using intelligent medical image understanding. Medical image classification, segmentation, detection and localization are the important tasks in medical image understanding.

\subsection{Medical image classification}

Medical image classification involves determining and assigning labels to medical images from a fixed set. The task involves the extraction of features from the image, and assigning labels using the extracted features. Let $I$ denote an image made of pixels and $c_{1}, c_{2}, \ldots, c_{r}$ denote the labels. For each pixel $x$, a feature vector $\zeta$, consisting of values $f\left(x_{i}\right)$ is extracted from the neighborhood $N(x)$ using (1), where $x_{i} \in N(x)$ for $i=0,1, \ldots, k$.

$\zeta=\left(f\left(x_{0}\right), f\left(x_{1}\right), \ldots, f\left(x_{k}\right)\right)$

A label from the list of labels $c_{1}, c_{2}, \ldots, c_{r}$ is assigned to the image based on $\zeta$.

\subsection{Medical image segmentation}

Medical image segmentation helps in image understanding, feature extraction and recognition, and quantitative assessment of lesions or other abnormalities. It provides valuable information for the analysis of pathologies, and subsequently helps in diagnosis and treatment planning. The objective of segmentation is to divide an image into regions that have strong correlations. Segmentation involves dividing the image $I$ into a finite set of regions $R_{1}, R_{1}, \ldots, R_{S}$ as expressed in (2).

$$
I=\bigcup_{i=1}^{s} R_{i}, R_{i} \cap R_{j}=\emptyset \text { and } i \neq j .
$$

\subsection{Medical image localization}

Automatic localization of pathology in images is quite an important step towards automatic acquisition planning and post imaging analysis tasks, such as segmentation and functional analysis. Localization involves predicting the object in an image, drawing a bounding box around the object and labeling the object.

The localization function $f(I)$ on an image $I$ computes $c, l_{x}, l_{y}, l_{w}, l_{h}$, which represent respectively, class label, centroid $x$ and $y$ coordinates, and the proportion of the bounding box with respect to width and height of the image as expressed in (3).

$f(I)=\left(c, l_{x}, l_{y}, l_{w}, l_{h}\right)$.

\subsection{Medical image detection}

Image detection aims at the classification and the localization of regions of interest by drawing bounding boxes around multiple regions of interest and labeling them. This helps in determining the exact locations of different organs and their orientation. Let $I$ be an image with $n$ objects or regions of interest. Then detection function $D(I)$ computes $c_{i}, x_{i}, y_{i}, w_{i}, h_{i}$ and these are respectively the class label, centroid $x$ and $y$ coordinates, proportion of the bounding box with respect to width and height of the image $I$ as given in the (4)

$\bigcup_{i=1}^{n} c_{i}, x_{i}, y_{i}, w_{i}, h_{i}=D(I)$.

\subsection{Evaluation metrics for image understanding}

There are many metrics used for the evaluation of performance of medical image understanding algorithms. The confusion matrix, also known as the error matrix, is the table used for visualizing the performance of an algorithm and for calculation of various evaluation metrics. It provides an insight about the types of errors that are made by the classifier. It is a square matrix in which rows represent the instances of actual results and the columns represent the instances of predicted results of the algorithm. The confusion matrix of a binary classifier is shown in Table 1 .

Here, $T_{P}$ indicates correctly identified positives, $T_{N}$ indicates correctly identified negatives, $F_{P}$ indicates incorrectly identified positives and $F_{N}$ indicates incorrectly identified negatives. $F_{P}$ is also known as false error and $F_{N}$ is known 
Table 1 Confusion matrix

\begin{tabular}{lll}
\hline Total test samples & Predicted positive & Predicted negative \\
\hline Actual positive & True positives $\left(T_{P}\right)$ & False negatives $\left(F_{N}\right)$ \\
Actual negative & False positives $\left(F_{P}\right)$ & True negatives $\left(T_{N}\right)$ \\
\hline
\end{tabular}

as miss. The sum of correct and incorrect predictions is represented as $T$ and expressed as in (5).

$T=T_{P}+T_{N}+F_{P}+F_{N}$.

Performance metrics can be determined with the help of confusion matrix and are given in Table 2.

\section{A brief introduction to CNNs}

Image understanding by animals is a very fascinating process, and a very simple task for them. But for a machine, to understand an image, there are lot of hidden complexities during the process. What animals feel is the eyes capturing the image, which is processed by the neurons and sent to the brain for interpretation. $\mathrm{CNN}$ is a deep learning algorithm inspired by the visual cortex of animal brain [30] and aims to imitate the visual machinery of animals. CNNs represents a quantum leap in the field of image understanding, involving image classification, segmentation, localization, detection etc. The efficacy of CNNs in image understanding is the main reason of its abundant use. CNNs are made of convolutions having learnable weights and biases similar to neurons (nerve cells) of the animal. Convolutional layers, activation functions, pooling and fully-connected layers are the core building blocks of CNNs, as depicted in Fig. 1. Very brief introduction to $\mathrm{CNNs}$ has been presented in this paper. Detailed discussions on CNNs are presented in $[9,41]$.

\subsection{Convolution layers (Conv layers)}

The visual cortex of the animal brain is made of neuronal cells which extract features of the images. Each neuronal cell extracts different features, which help in image understanding. The conv layer is modeled over the neuronal cells and its objective is to extract features, such as edges, colors,

Table 2 Performance evaluation metrics for image processing

\begin{tabular}{|c|c|c|c|}
\hline Evaluation metric & To determine & Formula & Preferred value \\
\hline Accuracy & Overall how often the result is true & $\frac{T_{P}+T_{N}}{T}$ & $\operatorname{High}(\approx 1)$ \\
\hline Misclassification result (error rate) & Overall how often the result is false & $\frac{F_{P}-F_{N}}{T}$ & Low $(\approx 0)$ \\
\hline True positive rate $\left(T_{P R}\right)$ (recall or sensitivity) & When it is actually true, how often does it predict true & $\frac{T_{P}}{F_{N}+T_{P}}$ & $\operatorname{High}(\approx 1)$ \\
\hline False positive rate $\left(F_{P R}\right)$ & When its actually false, how often does it predict true & $\frac{F_{P}}{F_{P}+T_{N}}$ & Low $(\approx 0)$ \\
\hline False negative rate $\left(F_{N R}\right)$ & & $\frac{F_{N}}{F_{N}+T_{P}}$ & Low $(\approx 0)$ \\
\hline Specificity & When its actually false, how often does it predict false & $\frac{T_{N}}{F_{P}+T_{N}}$ & $\operatorname{High}(\approx 1)$ \\
\hline Precision (positive predictive value $\left(P_{P V}\right)$ ) & When it predicts true, how often is it correct? & $\frac{T_{P}}{F_{P}+T_{P}}$ & $\operatorname{High}(\approx 1)$ \\
\hline F-Score (Dice similarity coefficient) & Harmonic mean of recall and precision. & $2 \times \frac{P_{P V} \times T_{P R}}{P_{P V}+T_{P R}}$ & $\operatorname{High}(\approx 1)$ \\
\hline Receiver operating characteristic (ROC) & $\begin{array}{l}\text { Commonly used graph to summarizes the perfor- } \\
\text { mance of a classifier over all possible thresholds. }\end{array}$ & Plot of recall verses FPR & $\operatorname{High}(\approx 1)$ \\
\hline Area under ROC (AUC) & The area under ROC & Plots $T_{P R}$ versus $F_{P R}$ & $\operatorname{High}(\approx 1)$ \\
\hline
\end{tabular}

Fig. 1 Building blocks of a $\mathrm{CNN}$
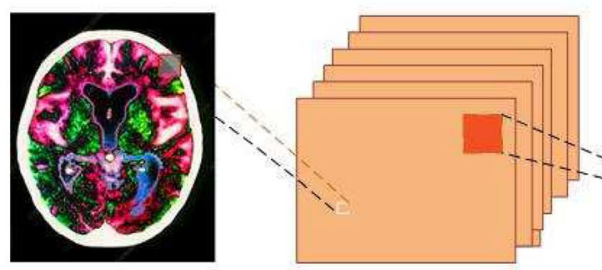

Input image

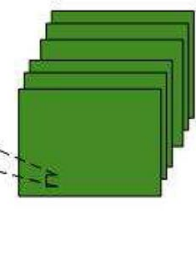

Pooling layers

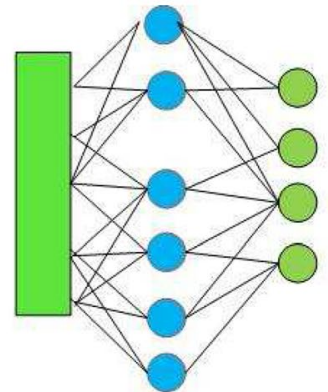

Output 
texture and gradient orientation. Conv layers are made of learnable filters called convolutional filters, or kernels, of size $n \times m \times d$, where $d$ is the depth of the image. During the forward pass, the kernels are convolved across the width and height of input volume and dot product is computed between the entries of the filter and the input. Intuitively, the CNN learns filters that gets activated when they come across edge, colors, texture etc. The output of the conv layer is fed into an activation function layer.

\subsection{Activation functions or nonlinear functions}

Since data in real world is mostly nonlinear, activation functions are used for nonlinear transformation of the data. It is used to ensure that the representation in the input space is mapped to a different output space as per the requirements. The different activation functions are discussed in Sects. 3.2.1-3.2.3.

\subsubsection{Sigmoid}

It takes a real-valued number $x$ and squashes it into range between 0 and 1 . In particular, large negative and positive inputs are placed very close to 0 and unity, respectively. It is expressed as in (6).

$f(x)=\frac{1}{1+e^{-x}}$.

\subsubsection{Tan hyperbolic}

It takes a real valued number $x$ and squashes it between -1 to 1 as expressed in (7).

$f(x)=\frac{1-e^{-2 x}}{1+e^{-2 x}}$.

\subsubsection{Rectified linear unit (ReLU)}

This nonlinear function takes a real valued number $x$ and converts $x$ to 0 if $x$ is negative. ReLU is the most often used nonlinear function for $\mathrm{CNN}$, takes less computation time and hence faster compared to the other two and is expressed in (8).

$f(x)=\max (0, x)$.

\subsection{Pooling}

Pooling layer performs a nonlinear down sampling of convolved feature. It decreases the computational power required to process the data through dimensionality reduction. It reduces the spatial size by aggregating data over space or feature type, controls overfitting and overcomes translation and rotational variance of images. Pooling operation results in partitioning of its input into a set of rectangle patches. Each patch gets replaced by a single value depending on the type of pooling selected. The different types are maximum pooling and average pooling.

\subsection{Fully connected (FC) layer}

FC layer is similar to artificial neural network, where each node has incoming connections from all the inputs and all the connections have weights associated with them. The output is sum of all the inputs multiplied by the corresponding weights. FC layer is followed by sigmoid activation function and performs the classifier job.

\subsection{Data preprocessing and augmentation}

The raw images obtained from imaging modalities need to be preprocessed and augmented before sending to CNN. The raw image data might be skewed, altered by bias distortion [55], having intensity inhomogeneity during capture, and hence needs to be preprocessed. Multiple data preprocessing methods exist and the preferred methods are mean subtraction and normalization. CNN needs to be trained on a larger dataset to achieve the best performance. Data augmentation increases the existing set of images by horizontal and vertical flips, transformations, scaling, random cropping, color jittering and intensity variations. The preprocessed, augmented image data is then fed into CNN.

\subsection{CNN architectures and frameworks}

Many CNN architectures have been proposed by researchers depending on kind of task to be performed. A few award-winning architectures are listed in Table 3. CNN frameworks (toolkits) enable the efficient development and implementation of deep learning methods. Various frameworks used by researchers and developers is listed in Table 4.

Table 3 Various award winning CNN architectures

\begin{tabular}{llll}
\hline Architecture & References & Architecture & References \\
\hline LeNet-5 & {$[43]$} & VGGNet & {$[70]$} \\
AlexNet & {$[41]$} & GoogLeNet & {$[3]$} \\
Overfeat & {$[65]$} & ResNet & {$[26]$} \\
ZFNet & {$[79]$} & Xception & {$[12]$} \\
\hline
\end{tabular}




\section{CNN applications in medical image classification}

\subsection{Lung diseases}

Interstitial lung disease (ILD) is the disorder of lung parenchyma in which lung tissues get scarred leading to respiratory difficulty. High resolution computed tomography (HRCT) imaging is used to differentiate between different types of ILDs. HRCT images have a high visual variation between different classes and high visual similarity within the same class. Therefore, accurate classification is quite challenging.

\subsubsection{Ensemble CNN}

Ensemble of rf and overfeat for classification of pulmonary peri fissural nodules of lungs was proposed in [14]. The complexity of the input was reduced by extracting twodimensional views from three-dimensional volume. The performance was enhanced by using a combination of overfeat followed by $\mathrm{rf}$. The bagging technique of $\mathrm{rf}$ boosted the performance of the model. The proposed model obtained an AUC of $86.8 \%$.

\subsubsection{Small-kernel CNN}

Low level textual information and more non linear activations enhances performance of classification was emphasized by [4]. The authors shrinked the kernel size to $2 \times 2$ to involve more non linear activations. The receptive fields were kept smaller to capture low level textual information. Also, to handle increasing complexity of the structures, the number of kernels were made proportional to the number of receptive field of its neurons. The model classified the lung tissue image into seven classes (a healthy tissue and six different ILD patterns). The results were compared against AlexNet and VGGNet and the ROC curves. The structure took only $20 \mathrm{~s}$ to classify the whole lung area in 30 slices of an average size HRCT scan image. AlexNet and VGG-Net took $136 \mathrm{~s}$ and $160 \mathrm{~s}$ for classification. The model delivered a classification accuracy of $85 \%$, while the traditional methods delivered an accuracy of $78 \%$.

\subsubsection{Whole image CNN}

Smaller image patches to prevent loss of spatial information and different attenuation ranges to enhance better visibility was proposed in [18]. Since the images were RGB, the proposed CNN model used three lung attenuation ranges namely, lower attenuation, normal attenuation and higher attenuation. To avoid overfitting, the images were augmented by jitter and cropping. A simple Alexnet model with the above variations was implemented and compared against other CNN models implemented to work on image patches. The performance metrics were accuracy and F-score. The model obtained an F-score of $100 \%$ and the average accuracy of $87.9 \%$.

\subsubsection{Multicrop pooling CNN}

Limitation of reduced training samples can be overcome by extraction of salient multi scale features. The features were extracted using multicrop pooling for automatic lung nodule malignancy suspicious classification in [68]. The model was a simple 3 layered CNN architecture but with multicrop pooling and randomized leaky ReLu as activation. The proposed method obtained accuracy and AUC of $87.4 \%$ and 93\%. Fivefold cross validation was used for evaluation.

The CNN applications in lung classification is summarized in Table 5.

\subsection{Coronavirus disease 2019 (COVID-19)}

COVID-19 is a global pandemic disease spreading rapidly around the world. Reverse Transcription Polymerase Chain Reaction (RT-PCR) is a commonly employed test for detection of COVID-19 infection. RT-PCR testing is the gold standard for COVID-19 testing, RT-PCR is very complicated, time-consuming and labor-intensive process, sparse availability and not very accurate. Chest $\mathrm{X}$-ray could be used for the initial screening of the COVID-19 in places having shortage of RT-PCR kits and is more accurate at diagnosis. Many researchers have used deep learning to classify if the chest infection is due to COVID-19 or other ailments.

\subsubsection{Customized CNN}

One of the initial model proposed for detection of COVID19 was a simple pretrained AlexNet model proposed in [45] and fine tuned on chest X-ray images. The results were very promising with accuracy of classifying positive and negative patients of around $95 \%$. Use of pretrained with transfer learning ResNet and InceptionNet CNN models were also proposed. These models demonstrated that the transfer learning models were also efficient and achieved a test accuracy of $93 \%$.

\subsubsection{Bayesian CNN}

Uncertainty was explored to enhance the diagnostic performance of classification of COVID-19 datasets in [22]. The primary aim of proposed method was to avoid COVID19 misdiagnoses. The method explored Monte-Carlo 


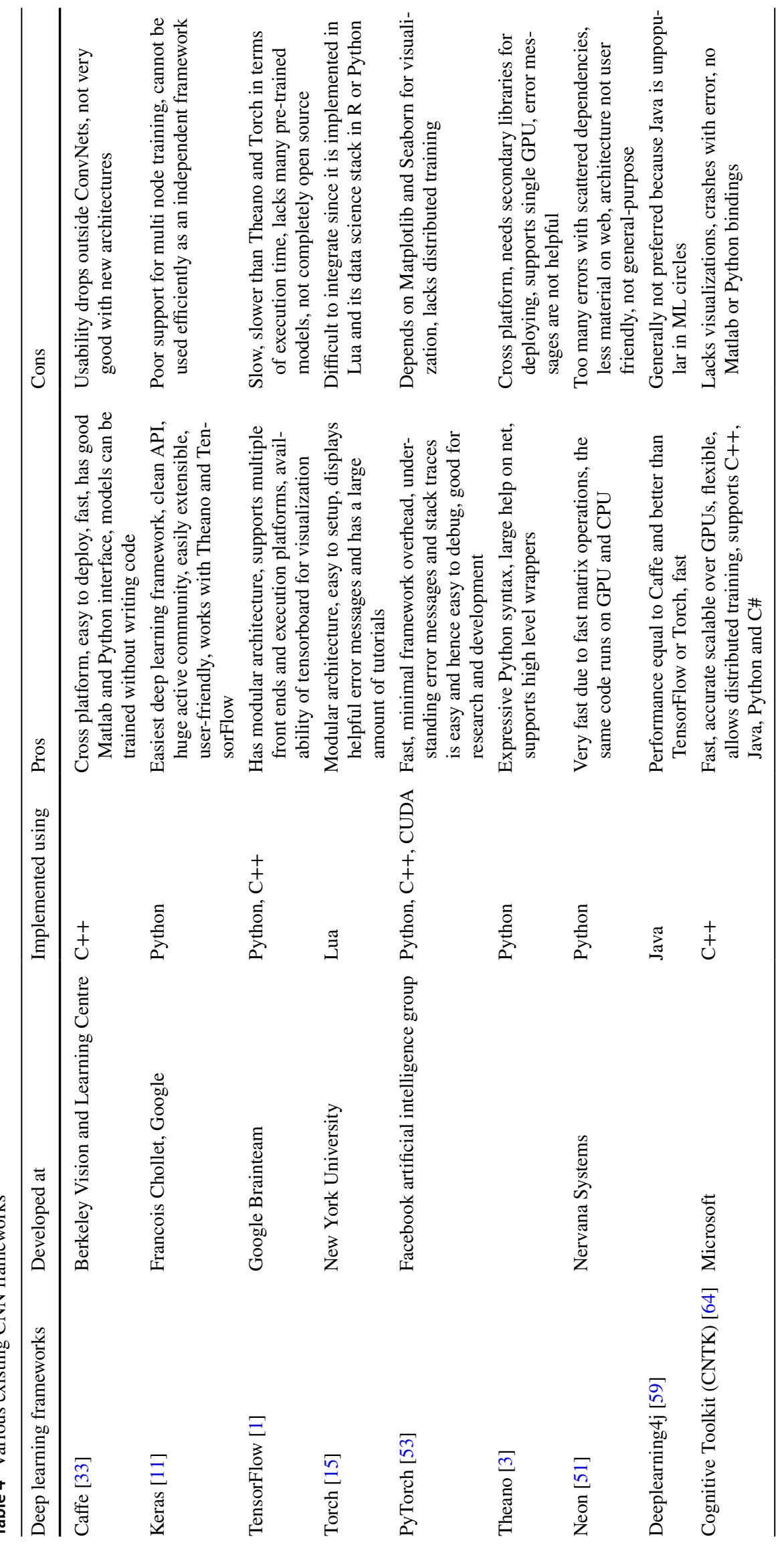


Table 5 A summary of CNN applications in ILD image classification surveyed in Sect. 4

\begin{tabular}{|c|c|c|c|c|c|}
\hline References & Preprocessing & Architecture & Dataset & Comparison against & Performance metric \\
\hline [44] & $\begin{array}{l}\text { Normalized with unit } \\
\text { variance and zero- } \\
\text { mean }\end{array}$ & $\begin{array}{l}\text { Conv, ReLU, maxpool, } \\
\text { FC } \times 3 \text {, backpropaga- } \\
\text { tion, batch processing, } \\
\text { dropout of } 0.6\end{array}$ & ILD & $\begin{array}{l}\text { SIFT, LBP, unsuper- } \\
\text { vised feature learning } \\
\text { using RBM }\end{array}$ & Recall, precision \\
\hline [4] & $\begin{array}{l}\text { Augmentation by rotat- } \\
\text { ing }\end{array}$ & $\begin{array}{l}\text { (Conv, Leaky ReLU, } \\
\text { avgpool) } \times 5 \text {, three FC, } \\
\text { softmax classifier }\end{array}$ & $\begin{array}{l}\text { ILD from University } \\
\text { of Geneva and Bern } \\
\text { University hospital }\end{array}$ & AlexNet, VGGNet & Time, ROC, AUC \\
\hline [18] & $\begin{array}{l}\text { Augmentation by adding } \\
\text { jitter and cropping }\end{array}$ & AlexNet architecture & Public ILD database & $\begin{array}{l}\text { CNN patch based } \\
\text { methods }\end{array}$ & $\begin{array}{l}\text { Accuracy } 87.9 \% \text { com- } \\
\text { pared to } 86.1 \% \text { using } \\
\text { patch based methods }\end{array}$ \\
\hline [68] & Augmentation & $\begin{array}{l}\text { (Conv, ReLU, maxpool } \\
\text { with multicrop) } \times 3+ \\
\text { FC, softmax }\end{array}$ & LIDC-IDRI dataset & HOG, LBP & $\begin{array}{l}\text { Accuracy } 87.4 \% \text { and AUC } \\
93 \%\end{array}$ \\
\hline
\end{tabular}

Dropweights Bayesian CNN to estimate uncertainty in deep learning, to better the diagnostic performance of human-machine decisions. The method showed that there is a strong correlation between classification accuracy and estimated uncertainty in predictions. The proposed method used ResNet50v2 model. The softmax layer was preceded by dropweights. Dropweights were applied as an approximation to the Gaussian Process, which was used to estimate meaningful model uncertainty. The softmax layer finally outputs each possible class label's probability distribution.

\subsubsection{PDCOVIDNET}

Use of dilation to detect dominant features in the image was explored in [13]. The authors proposed parallel dilated $\mathrm{CNN}$ model. The dilated module involved skipping of pixels during convolution process. Parallel CNN branches are proposed with different dilation rates. The features obtained from parallel branches were concatenated and input to the next convolution layer. Concatenation-convolution operation was used to explore feature relationship of dilated convolutions so as to detect dominant features for classification. The model also used Grad-CAM and Grad-CAM++ to highlight the regions of class-discriminative saliency maps. The performance metrics used were accuracy, precision, recall, F1-score with ROC/AUC and are $96.58 \%, 95 \%, 91 \%, 93 \%$ and 0.991 respectively.

\subsubsection{CVR-Net}

To prevent degrading of final prediction and to compensate for lesser number of datasets, multi scale multi encoder ensemble CNN model for classification of COVID-19 was proposed in [24]. The proposed model ensembled feature maps at different scales obtained from different encoders. To avoid overfitting, geometry based image augmentations and transfer learning was proposed. To overcome vanishing gradients, each encoder consisted of residual and convolutional blocks to allow gradients to pass, like in resNet architecture. Moreover Depth-wise separable convolution was used to create a light weight network. The depth information of feature map was enhanced by concatenating different 2D feature maps of different encoders in channel-wise. The performance metrics for classifying images into positive and negative were recall, precision, f1-score and accuracy. The model showed a very efficient performance with score of nearly $98 \%$ for all the metrics.

\subsubsection{Twice transfer learning CNN}

A denseNet model trained twice using transfer learning approach was proposed in [6]. The denseNet201 model was trained initially on imageNet dataset, followed by chest X-ray 14 dataset and then fine tuned on COVID19 dataset. Various combinations of training the model first with single transfer learning, twice transfer learning, twice transfer learning with output neuron keeping were experimented. The model with twice transfer learning with output neuron keeping achieved the best performance accuracy of $98.9 \%$ over the other models. Transfer learning on chest X-ray 14 dataset enhanced the result, as the model had learnt most of the features related to chest abnormalities.

\subsection{Immune response abnormalities}

Autoimmune diseases result from an abnormal immune response to a normal body part. The immune system of the body attacks the healthy cells in such diseases. Indirect immunofluorescence (IIF) on human epithelial-2 (HEp-2) cells is used to diagnose an autoimmune disease. Manual identification of these patterns is a time-consuming process. 


\subsubsection{CUDA ConvNet CNN}

Preprocessing using histogram equalization and zero-mean with unit variance increases classification accuracy by an additional $10 \%$ with augmentation was proposed in [7]. The experiments also demonstrate that pretraining, followed by fine tuning boosts performance. It achieved an average classification accuracy of $80.3 \%$ which was greater than the previous best of $75.6 \%$. The authors used Caffe library [33] and CUDA ConvNet model architecture to extract CNN-based features for classification of HEp-2 cells.

\subsubsection{Six-layer CNN}

Preprocessing and augmentation enhanced the mean classification accuracy of HEp-2 cell images and was shown in [21]. The framework consisted of three stages of image preprocessing, network training and feature extraction with classification. Mean classification accuracy of $96.7 \%$ on ICPR-2012 dataset was obtained. The CNN approaches for HEp- 2 cell classification are summarized in Table 6.

\subsection{Breast tumors}

Breast cancer is the most common cancer that affects women across the world. It can be detected by the analysis of mammographs. Two radiologists independently reading the same mammogram has been advocated to overcome any misjudgement.

\subsubsection{Stopping monitoring CNN}

Stopping monitoring to reduce the computation time is proposed in [52]. Stopping monitoring was performed using AUC on validation set. The model extracted region of interest (ROI) by cropping. The images were augmented to increase the number of samples and also to prevent overfitting. The CNN model proposed was for classification of breast tumors. The result was compared against the stateof-the-art image descriptors HOG and HOG divergence.
The proposed method resulted in AUC of $82.2 \%$ compared to $78.7 \%$ for other methods.

\subsubsection{Ensemble CNN}

Fine tuning enhanced the performance in the case of limited dataset was proposed in [34]. The proposed model was similar to AlexNet and was pretrained on imagenet, followed by fine tuning on breast images due to shortage of breast images. The middle and high level features were extracted from different layers of the network and fed into SVM classifiers for training. The model classified the breast masses into malignant and benign. Due to extraction of efficient features by deep network, simple classifier also resulted in accuracy of $96.7 \%$. The proposed method was compared against bag-of-words, HOG and SIFT and it outperformed all of them.

\subsubsection{Semi-supervised CNN}

CNN can also be used in scenarios involving sparse labeled data and abundant unlabeled data. To overcome the sparse labeled data problem, a new graph based semi supervised learning techniques for breast cancer diagnosis was proposed in [73]. For removal of redundancies and feature correlations, dimensionality reduction was employed. The method used four modules, feature extraction to extract 21 features from breast masses, data weighing to minimize the influence of noisy data and division of co-training data labeling followed by the $\mathrm{CNN}$. It involved sub patches extraction of ROIs which were input to three pairs of conv layers, maxpooling and FC layer. Three models CNN, SVM and ANN are compared. The AUC for $\mathrm{CNN}$ for mixture of labeled and unlabeled data was $88 \%$ compared to $85 \%$ of SVM and $84 \%$ of ANN and accuracy for CNN with mixed data was $82 \%$ compared to $80 \%$ for SVM and $80 \%$ for ANN. The CNN approaches for breast tumor classification are summarized in Table 7.

Table 6 A summary of CNN applications in HEp-2 cell classification surveyed in Sect. 4

\begin{tabular}{|c|c|c|c|c|c|}
\hline References & Preprocessing & Architecture & Dataset & Comparison against & Perfromance metric \\
\hline [7] & $\begin{array}{l}\text { Augmented by affine transform } \\
\text { and intensity variations, } \\
\text { green channel images used }\end{array}$ & $\begin{array}{l}\text { Conv, maxpool, ReLU, conv, } \\
\text { avgpool, ReLU, conv, } \\
\text { avgpool, FC with dropout, } \\
\text { weight decay, softmax with } \\
\text { multinomial logistic regres- } \\
\text { sion }\end{array}$ & ICPR 2014 & $\begin{array}{l}\text { ICPR } 2012 \text { benchmark and } \\
\text { winners model }\end{array}$ & $\begin{array}{l}\text { Average classifica- } \\
\text { tion accuracy } 81 \%\end{array}$ \\
\hline [21] & $\begin{array}{l}\text { Normalized using zero mean } \\
\text { and unit variance, and } \\
\text { rotated }\end{array}$ & $\begin{array}{l}(\text { Conv, tanh, maxpool }) \times 3 \text {, } \\
\text { FC } \times 2 \text {, softmax regression, } \\
\text { outputs six classes }\end{array}$ & ICPR 2014 & $\begin{array}{l}\text { ICPR } 2012 \text { classification } \\
\text { contestants }\end{array}$ & Accuracy $96.17 \%$ \\
\hline
\end{tabular}


Table 7 A summary of CNN applications in breast medical image classification surveyed in Sect. 4

\begin{tabular}{|c|c|c|c|c|}
\hline References & Preprocessing & Architecture & Comparison against & Performance metric \\
\hline [34] & $\begin{array}{l}\text { Normalized by subtracting } \\
\text { mean, dividing by standard } \\
\text { deviation, augmented by } \\
\text { flipping }\end{array}$ & $\begin{array}{l}(\text { Conv, ReLU, maxpool }) \times 5, \\
(\mathrm{FC}+\mathrm{ReLU}) \times 2+\mathrm{FC}, \text { sto- } \\
\text { chastic gradient descent }\end{array}$ & BOW, HOG, SIFT, VGGNet & ROC and accuracy $96.7 \%$ \\
\hline [52] & $\begin{array}{l}\text { Crop, augmentation by flipping } \\
\text { and rotating, global contrast } \\
\text { normalization, local contrast } \\
\text { normalization }\end{array}$ & $\begin{array}{l}\text { (Conv, ReLU, maxpool) } \times 2+ \\
\text { FC }\end{array}$ & HOG, HGD & AUC $82.2 \%$ \\
\hline [73] & $\begin{array}{l}\text { Feature extraction, data weigh- } \\
\text { ing, data labeling }\end{array}$ & $($ Conv, maxpool $) \times 3$, FC & $\begin{array}{l}\text { SVM, ANN, labeled data, unla- } \\
\text { beled data and mixed data }\end{array}$ & Accuracy $82.43 \%$, AUC $88.18 \%$ \\
\hline [78] & $\begin{array}{l}\text { Image oversegmented into } \\
\text { atomic regions using super- } \\
\text { pixel based scheme }\end{array}$ & $\begin{array}{l}(\text { Conv, maxpool }) \times 2, F C \times 2, \\
\text { softmax classifier }\end{array}$ & $\begin{array}{l}\text { Linder, Bianconi, } \\
\text { DCNN-Ncut + SVM, } \\
\text { DCNN-Ncut + SMC, } \\
\text { DCNN-Ncut + SVM, } \\
\text { DCNN-SLIC-SVM, DCNN- } \\
\text { SLIC-SMC }\end{array}$ & ROC, AUC, accuracy \\
\hline
\end{tabular}

\subsection{Heart diseases}

Electrocardiogram (ECG) is used for the assessment of the electrical activity of the heart to detect anomalies in the heart.

\subsubsection{One-dimensional CNN}

ECG classification using CNN model demonstrates superior performance with classification accuracy of $95 \%$ was proposed in [40]. The model comprised of one-dimensional CNN with three conv layers and two FC layers that fused feature extraction and classification into a single learning body. Once the dedicated $\mathrm{CNN}$ was trained for a particular patient, it could be solely used to classify ECG records in a fast and accurate manner.

\subsubsection{Fused CNN}

Classification of echocardiography videos require both spatial and temporal data. Fused CNN architecture using both spatial and temporal data was proposed in [20]. It used a two-path CNN, one along the spatial direction and the other along temporal direction. Each individual CNN path executed individually and was fused only after obtaining the final classification scores. The spatial CNN learnt the spatial information automatically from the original normalised echo video images. Temporal CNN learnt from acceleration images along the time direction of the echo videos. The outputs of both CNNs were fused and applied to softmax classifier for the final classification. The proposed model achieved an average accuracy of $92 \%$ compared to $89.5 \%$ for single path CNN, $87.9 \%$ for three-dimensional KAZE, $73.8 \%$ for three-dimensional SIFT. The long time required for initial training was the disadvantage of this approach. The CNN approaches to heart classification are summarized in Table 8.

\subsection{Eye diseases}

\subsubsection{Gaussian initialized CNN}

Initial training time can be reduced by Gaussian initialization, and overfitting can be avoided by weighted class weights. This was proposed for classifying diabetic retinopathy (DR) in fundus imagery in [57]. The performance was compared with SVM and other methods that required feature extraction prior to classification. The method achieved $95 \%$ specificity but less sensitivity of $30 \%$. The trained CNN did a quick diagnosis and gave an immediate response to the patient during screening.

\subsubsection{Hyper parameter tuning inception-v4}

Automated hyper parameter tuning inception-v4 (HPTIv4) model for DR in color fundus images classification and detection is proposed in [67]. The images are preprocessed using CLAHE to enhance contrast level, segmented using histogram based segmentation model. Hyper parameter tuning is done using Bayesian optimization method, as Bayesian model has the ability to analyze the previous validation outcome, to create a probabilistic model. Classification is done using HPTI-v4 model followed by multi layer perceptron. The classification is applied on MESSIDOR DR dataset. The CNN model performance was extraordinary with the accuracy, sensitivity, and specificity of $99.49 \%, 98.83 \%$, and $99.68 \%$ respectively. 
Table 8 A summary of CNN applications in heart classification surveyed in Sect. 4

\begin{tabular}{|c|c|c|c|c|c|}
\hline References & Preprocessing & Architecture & Dataset & Comparison against & Performance metric \\
\hline [40] & $\begin{array}{l}\text { ECG signals filtered using } \\
\text { bandpass filter at } 0.1-100 \\
\mathrm{~Hz} \text { and digitized at } 360 \\
\mathrm{~Hz} .\end{array}$ & $\begin{array}{r}\text { One-dimensional } \mathrm{CNN} \\
\text { with conv } \times 3, \mathrm{FC} \times 2\end{array}$ & $\begin{array}{l}\text { MIT/BIH arrhythmia } \\
\text { database }\end{array}$ & Other existing methods & $\begin{array}{l}\text { Accuracy, sensitiv- } \\
\text { ity, specificity }\end{array}$ \\
\hline [20] & $\begin{array}{l}\text { Image resized to } \\
175 \times 200 \times 26\end{array}$ & $\begin{array}{l}\text { Two path CNN of seven } \\
\text { layers (conv, ReLU, } \\
\text { maxpool) } \times 2 \text {, (conv, } \\
\text { ReLU) } \times 2 \text {, (conv, ReLU, } \\
\text { maxpool), (conv, drop- } \\
\text { out) } \times 2\end{array}$ & $\begin{array}{l}\text { Tsinghua University } \\
\text { Hospital, Beijing and } \\
\text { Fuzhou University Hos- } \\
\text { pital, China }\end{array}$ & SIFT, KAZE & Accuracy $92.1 \%$ \\
\hline
\end{tabular}

\subsection{Colon cancer}

\subsubsection{Ensemble CNN}

Usage of small patches increased the amount of training data and localized the analysis to small nuclei in images. This enhanced the performance of detecting and classifying nuclei in H\&E stained histopathology images of colorectal adenocarcinoma. This was proposed in [71]. The model also demonstrated locality sensitive deep learning approach with neighboring ensemble predictor (NEP) in conjunction with a standard softmax CNN and eliminated need of segmentation. The model used dropout to avoid overfitting. The model obtained an AUC of $91.7 \%$ and F-score of $78.4 \%$

The CNN approaches for colon cancer classification are summarized in Table 9.

\subsection{Brain disorders}

Alzheimer's disease causes the destruction of brain cells leading to memory loss. Classification of Alzheimer's disease (AD) has been challenging since it involves selection of discriminative features.

\subsubsection{Fused CNN}

Fusion of two-dimensional CNN and three-dimensional CNN achieves better accuracy was demonstrated in [19]. Information along $\mathrm{Z}$ direction acts very crucial for analysis of brain images and three-dimensional CNN was used to retain this information. Since the thickness of brain CT images is thicker than MRI images, geometric normalization of CT images were performed. Output of the last conv layer of two-dimensional CNN was fused with three-dimensional convoluted data to get three classes (Alzheimer's, lesions, and healthy data). It was compared with two hand-crafted approaches SIFT and KAZE for accuracy and achieved better accuracy of $86.7 \%, 78.9 \%$ and $95.6 \%$ for $\mathrm{AD}$, lesion and normal class, respectively.

\subsubsection{Input cascaded CNN}

Lack of training data can be overcome by extensive augmentation and fine tuning was proposed in [62]. Multi-grade brain tumor classification was performed by segmenting the tumor regions from an MR image using input cascaded $\mathrm{CNN}$, extensive augmentation and then fine-tuned using data augmented. The performance was compared against state-ofart methods. It resulted in an accuracy of $94.58 \%$, sensitivity of $88.41 \%$ and specificity of $96.58 \%$.

Table 9 A summary of CNN applications in colon medical image classification surveyed in Sect. 4

\begin{tabular}{lllll}
\hline References & Preprocessing & Architecture & Comparison against & Performance metric \\
\hline [71] & Augmented using small patches & $\begin{array}{c}\text { NEP with standard softmax } \\
\text { CNN, ReLU, dropout }\end{array}$ & AUC 91.7\%, F-score 78.4\%. \\
{$[78]$} & $\begin{array}{c}\text { Image subdivided into } 80 \times 80 \\
\text { using sliding window, and } \\
\text { border padding to prevent } \\
\text { artifacts }\end{array}$ & $\begin{array}{c}\text { (Conv, maxpool }) \times 2, \text { FC } \times 2, \\
\text { softmax }\end{array}$ & $\begin{array}{c}\text { Linder, Bianconi, DCNN-SW- } \\
\text { SVM, DCNN-SW-SMC }\end{array}$ & ROC, AUC, accuracy \\
& $\begin{array}{l}\text { Normalized by subtracting } \\
\text { mean, dividing by standard } \\
\text { deviation, augmented by } \\
\text { flipping }\end{array}$ & $\begin{array}{c}\text { (Conv, pool) }) \times 2, \text { conv, FC with } \\
\text { dropout, softmax }\end{array}$ & $\begin{array}{c}\text { BFD, SSF, DT-CTW, MB-LBP, } \\
\text { SIFT }\end{array}$ & Accuracy 90.96\% \\
& & & \\
\end{tabular}


The CNN approaches for medical image classification discussed above are summarized in Table 10.

\section{CNN applications in medical image segmentation}

CNNs have been applied to implement efficient segmentation of images of brain tumors, hearts, breasts, retina, fetal abdomen, stromal and epithelial tissues.

\subsection{Brain tumors}

MRI is used to obtain detailed images of the brain to diagnose tumors. Automatic segmentation of a brain tumor is very challenging because it involves the extraction of high level features.

\subsubsection{Small kernel CNN}

Patch-wise training and use of small filter sizes $(3 \times 3)$ was proposed for segmentation of gliomas in [54]. This provided an advantage of deep architecture, while retaining the same receptive fields. Two separate models were trained for high and low gliomas. High glioma model consisted of eight conv layers and three dense layers. Low glioma model contained four conv layers and three dense layers. Maxpooling was used along with dropout for dense layers. It ranked fourth in the BRATS-2015 challenge. Data augmentation was achieved by rotation which enhanced the performance of segmentation of gliomas.

\subsubsection{Fully blown CNN}

Fully blown MRI two-dimensional images enhances performance of segmentation of sub-cortical human brain structure. This was shown in [66]. The proposed model applied Markov random field on CNN output to impose volumetric homogeneity to the final results. It outperformed several state-of-the-art methods.

\subsubsection{Multipath CNN}

Two pathways, one for convolution and the other for deconvolution, enhances segmentation output was shown in [8]. The model was used for automatic MS lesions segmentation. The model had convolutional pathway consisting of alternating conv, pool layers, and a deconvolutional pathway consisting of alternate deconv layer and unpooling layer. The pretraining was performed by convolutional RBMs (convRBM). Both pretraining and fine training were performed on a highly optimized GPU-accelerated implementation of three-dimensional convRBMs and convolutional encoder networks (CEN). It was compared with five publicly available methods and established as comparison reference points. The model performance was evaluated using evaluation metrics DSC, TPR and FPR. TPR and FPR achieved were comparatively better than the previous models developed. However, it achieved lesser DSC in comparison to other methods.

\subsubsection{Cascaded CNN}

In case of imbalanced label distributions, two phase training could be used. Global contextual features and local detailed features can be learned simultaneously by two-pathway

Table 10 A summary of CNN applications in medical image classification surveyed in Sect. 4

\begin{tabular}{|c|c|c|c|c|c|}
\hline References & Organ & Preprocessing & Architecture & Dataset & Performance metric \\
\hline [19] & Brain & $\begin{array}{l}\text { Geometric normalization } \\
\text { of CT images }\end{array}$ & $\begin{array}{c}\text { Conv } \times 5 \text {, (conv }+ \text { drop- } \\
\text { out }) \times 3 \text {, fusion with } \\
3-\mathrm{D} \text { convoluted data }\end{array}$ & $\begin{array}{l}\text { 3-D datasets from } 282 \\
\text { subjects ( } 51 \text { with AD, } \\
118 \text { with lesions, } 117 \\
\text { normal) }\end{array}$ & $\begin{array}{l}\text { Accuracy } 86.7 \%, 78.9 \% \text { and } \\
95.6 \% \text { for AD, lesion and } \\
\text { normal class }\end{array}$ \\
\hline [63] & Brain & $\begin{array}{l}\text { Motion correction, } \\
\text { skull stripping, spatial } \\
\text { smoothing }\end{array}$ & LeNet & ADNI dataset & Accuracy $96.86 \%$ \\
\hline$[62]$ & Brain & Data augmentation & $\begin{array}{l}\text { Input cascaded CNN for } \\
\text { segmentation, VGG-19 } \\
\text { with transfer learning }\end{array}$ & $\begin{array}{l}\text { Radiopedia and brain } \\
\text { tumor }\end{array}$ & $\begin{array}{l}\text { Accuracy } 94.58 \% \text {, sensitiv- } \\
\text { ity } 88.41 \% \text {, specificity } \\
96.58 \%\end{array}$ \\
\hline [57] & Eye, retinopathy & $\begin{array}{l}\text { Color normalization, } \\
\text { image size reduced to } \\
512\end{array}$ & $\begin{array}{l}\text { Conv } \times 10, \mathrm{FC} \times 3 \text { with } \\
\text { softmax classifier }\end{array}$ & Kaggle & $\begin{array}{l}\text { Specificity } 95 \% \text { but less } \\
\text { sensitivity } 30 \%\end{array}$ \\
\hline [82] & Digestive organs & & $\begin{array}{l}(\text { Conv, maxpool }) \times 3, \text { FC } \\
\times 2 \text {, mini batch stochastic } \\
\text { gradient descent }\end{array}$ & $\begin{array}{l}\text { Real clinical data of wire- } \\
\text { less capsule endoscopy } \\
\text { images }\end{array}$ & Accuracy $95 \%$ \\
\hline
\end{tabular}


architecture for brain segmentation and was proposed in [25]. The advantage of two-pathway was, it could recognize fine details of the tumor at a local scale and correct labels at a global scale to yield a better segmentation. Slice-byslice segmentation from the axial view due to less resolution in the third dimension was performed. The cascaded CNN achieved better rank than two-pathway CNN and was ranked second at the MICCAI BRATS-2013 challenge. The evaluation metrics used were DSC, specificity and sensitivity and the obtained values were $79 \%, 81 \%$ and $79 \%$. The time taken for segmentation was between $25 \mathrm{~s}$ and $3 \mathrm{~min}$.

\subsubsection{Multiscale CNN}

In case of brain tumor segmentation, a multiscale CNN architecture for extracting both local and global features at different scales was proposed in [80]. The model performed better due to different features extracted at various resolution. The computation time was reduced by exploiting a two-dimensional CNN instead of a three-dimensional CNN. Three patch sizes $48 \times 48,28 \times 28$ and $12 \times 12$ were input to three CNNs for feature extraction. All the features extracted were input to the FC layer. Evaluation of the model was by DSC and accuracy. The model performance was almost as stable as the best method with an accuracy of nearly $90 \%$.

\subsubsection{Multipath and multiscale CNN}

Twopath and multiscale architecture were also explored for brain lesion segmentation by [37]. The model exploited smaller kernels to get local neighbour information and employed parallel convolutional pathways for multiscale processing. It achieved highest accuracy when applied on patients with severe traumatic brain injuries. It could also segment small and diffused pathologies. Three-dimensional $\mathrm{CNN}$ produced accurate segmentation borders. FC threedimensional CRF imposed regularization constraints on $\mathrm{CNN}$ output and produced final hard segmentation labels. Also, due to its generic nature, it cold be applied to different lesion segmentation tasks with slight modifications. It was ranked first in the stroke lesions ISLES-SISS-2015 challenge.

Advantages of multipath and multiscale CNN was exploited for automatic segmentation of analytical brain images in [49]. The bigger kernel was used for spatial information. A separate network branch was used for each patch size, and only the output layer was shared. Mini batch learning and RMSprop were used to train the network with ReLU and cross entropy as the cost function. Automatic segmentation was evaluated using the DSC and mean surface distance between manual and automatic segmentation. It achieved accurate segmentation in terms of DSC for all tissue classes. The $\mathrm{CNN}$ approaches for brain segmentation discussed above are summarized in Table 11.

\subsection{Breast cancer}

Breast cancers can be predicted by automatically segmenting breast density and by characterizing mammographic textural patterns.

\subsubsection{FCNN}

Redundant computations in conv and max pool layers can be avoided by using ROI segmentation in a fast scanning deep CNN (FCNN). The above technique was applied for segmentation of histopathological breast cancer images and was proposed in [72]. The proposed work was compared against three texture classification methods namely raw pixel patch with large scale SVM, local binary pattern feature with large scale SVM and texton histogram with logistic booting. The evaluation metrics used were accuracy, efficiency and scalability. Ihe proposed method was robust to intra-class variance. It achieved an F-score of $85 \%$, whereas the other methods delivered the maximum F-score of $75 \%$. It took only $2.3 \mathrm{~s}$ to segment image of resolution of $1000 \times 1000$.

\subsubsection{Probability map CNN}

Probability maps were explored for iterative region merging for shape initialization along with compact nucleus shape repository with selection-based dictionary learning algorithm in [77]. The model resulted in better automatic nucleus segmentation using CNN. The framework was tested on three types of histopathology images namely, brain tumor, pancreatic neuro endocrine tumor and breast cancer. The parameters for comparison were precision, recall and F-score. It achieved better performance when compared to SVM, RF and DBN, especially for breast cancer images. Pixel-wise segmentation accuracy measured using DSC, HD and MAD resulted in superior performance when compared to other methods.

\subsubsection{Patch CNN}

Advantages of patch-based CNN was exploited in [78]. The method also exploited super pixel method to over segment breast cancer H\&E images into atomic images. The result was natural boundaries with errors being subtle and less egregious, whereas sliding window methods resulted in zigzag boundaries. Both patch-based CNN and superpixel techniques were combined for segmenting and classifying the stromal and epithelial regions in histopathological images for detection of breast and colorectal cancer. The proposed model outperformed CNN with SVM. The comparison was done against 


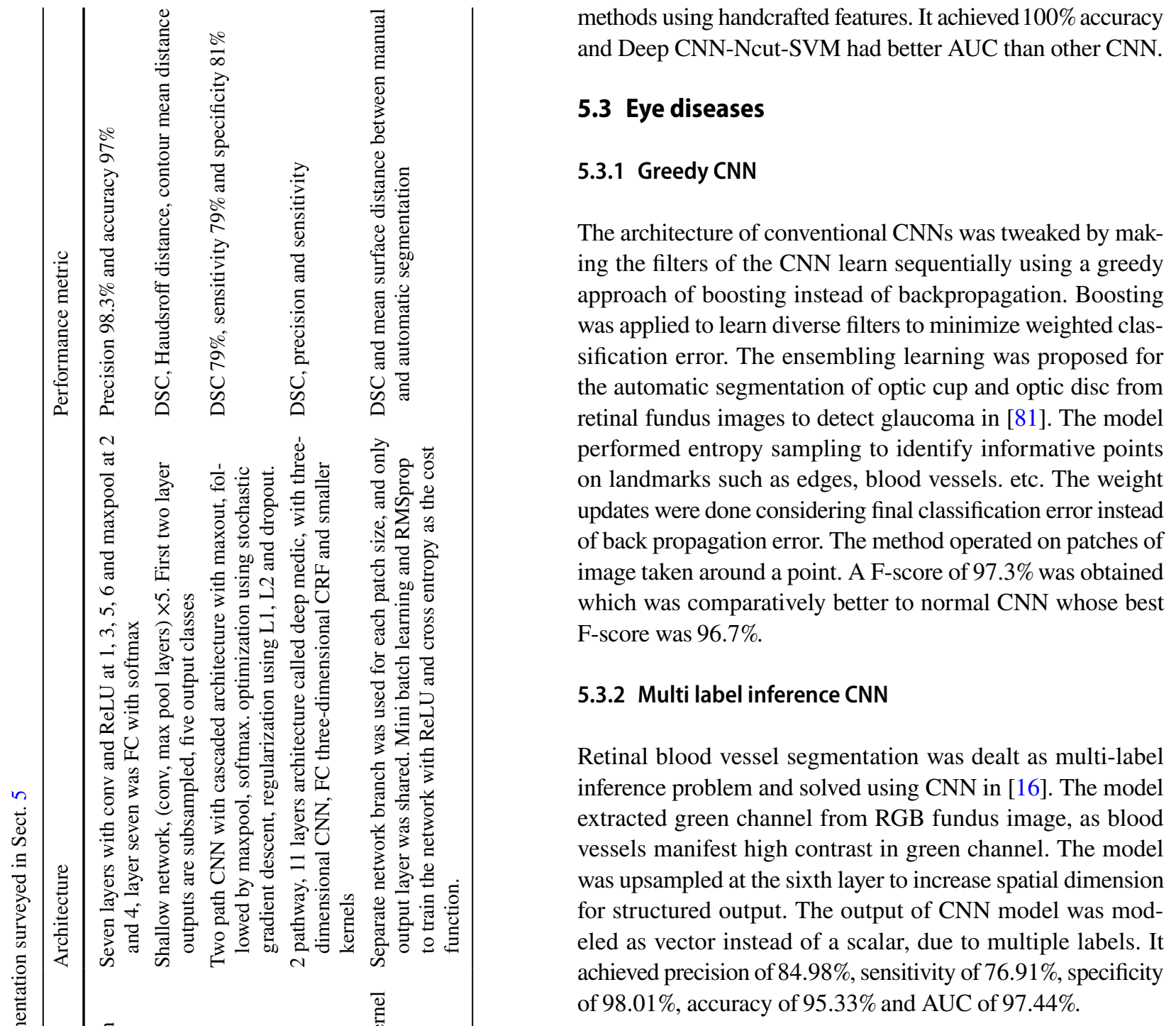

\subsection{Lung}

\subsubsection{U net}

Lung segmentation and bone shadow exclusion techniques for analysis of lung cancer using U-net architecture is proposed in [23]. The images were preprocessed to eliminate bone shadow and a simple U-net architecture was used to segment the lung ROI. The results obtained were very promising and showed a good speed and precise segmentation. The CNN approaches for medical image segmentation discussed above are summarized in Table 12. 
Table 12 A summary of CNN applications in medical image segmentation surveyed in Sect. 5

\begin{tabular}{|c|c|c|c|c|}
\hline References & Organ & Dataset & Comparison against & Performance metric \\
\hline [8] & MS lesion & $\begin{array}{l}\text { ISBI } 2015 \text { and MICCAI } 2008 \\
\text { datasets }\end{array}$ & Five publicly available methods & $\begin{array}{l}\text { DSC, TPR and FPR. Achieved better } \\
\text { TPR and FPR, but less DSC values }\end{array}$ \\
\hline [36] & Breast & $\begin{array}{l}\text { Dutch breast cancer screening } \\
\text { dataset }\end{array}$ & State of the art methods & AUC \\
\hline [72] & Breast & $\begin{array}{l}\text { The Cancer Genome Atlas breast } \\
\text { cancer dataset }\end{array}$ & $\begin{array}{l}\text { Three texture classification methods } \\
\text { namely RPLSVM, LBPLSVM and } \\
\text { THLB }\end{array}$ & $\begin{array}{l}\text { Accuracy, efficiency and scalability } \\
\text { and F-score of } 85 \%\end{array}$ \\
\hline [77] & Nucleus & $\begin{array}{l}\text { Brain tumor, pancreatic NET and } \\
\text { breast cancer }\end{array}$ & SVM, RF and DBN & Precision, recall and F-score \\
\hline [83] & Heart & CCTA scans of 60 patients & Manual segmentation & $\begin{array}{l}\text { Sensitivity } 95 \% \text { and specificity } 96.6 \% \\
\text { DSC } 85 \% \text { and mean absolute sur- } \\
\text { face distance } 85 \%\end{array}$ \\
\hline [81] & Eye glaucoma & DRISHTI-GS, Messidor & Manual & Precision, recall, F-score \\
\hline
\end{tabular}

\section{CNN applications in medical image detection}

\subsection{Breast tumors}

A Camelyon grand challenge for automatic detection of metastatic breast cancer in digital whole slide images of sentinel lymph node biopsies is organised by the International Symposium of Biomedical Imaging.

\subsubsection{GoogLeNet CNN}

The award-winning system with performance very closer to human accuracy was proposed in [76]. The computation time was reduced by first excluding the white background of digital images using Otsu's algorithm. The method exploited advantages of patch-based classification to obtain better results. Also the model trained extensively on misclassified image patches, to decrease classification error. The results of the patches were embedded on heatmap image and heatmaps were used to compute evaluation scores. AUC of $92.5 \%$ was obtained, and was the top performer in the challenge. In case of lesion based detection, the system achieved the sensitivity of $70.51 \%$, whereas the second ranking score was $57.61 \%$.

\subsection{Eye diseases}

\subsubsection{Dynamic CNN}

Random assignment of weights, speeds up the training and improves the performance. This was proposed for hemorrhage detection in fundus eye images in [75]. Also the samples were dynamically selected at every training epoch from a large pool of medical images. Pre-processing was performed using image contrast using gaussian filters. To prevent overfitting, the images were augmented. For correct classification of hemorrhage, the result was convolved with gaussian filter to smoothen the values. It achieved sensitivity, specificity and ROC of $93 \%, 91.5 \%$ and $98 \%$, whereas non selective sampling obtains sensitivity, specificity and ROC of $93 \%, 93 \%$ and $96.6 \%$ for Messidor dataset. AUC was used to monitor overfitting during training and when AUC value reached a stable maximum, the $\mathrm{CNN}$ training was stopped.

\subsubsection{Ensemble CNN}

An ensemble performs better than a single CNN and can be used to achieve higher performance. The ensemble model for detection of retinal vessels in fundus images is proposed in [46]. The model was an ensemble of twelve CNNs. Each CNN's output probability was averaged to get the final vessel's probability of each pixel. The probability was used to discriminate between vessel pixels from non-vessel ones for detection. The performance measures, accuracy and Kappa score were compared with existing state of the art methods. It stood second in terms of accuracy as well as in kappa score. The model obtained a FROC score of 0.928 .

\subsection{Cell division}

\subsubsection{LeNet CNN}

Augmentation and shifting the centroid of the object enhanced the performance, and was proposed in [69]. The model was for automatic detection of mitosis (cell divisions) and quantification of mitosis occurring during scratch assay. The positive example training samples was augmented by mirroring and rotating by $45^{\circ}$ and centering by shifting the centroid of the object to the patch center. A random additional sampling of negative samples was added in the same amount as positive examples. The performance parameters used were sensitivity, specificity, AUC and F-score 
and compared with SVM. The results indicated significant increase in F-score (for SVM 78\%, for CNN 89\%). The model concluded that both positive and negative samples are needed for better performance. The CNN applications in medical image detection reviewed in this paper are summarized in Table 13.

\section{CNN applications in medical image localization}

\subsection{Breast tumors}

\subsubsection{Semi-supervised deep CNN}

To overcome challenges of sparsely labeled data, a model was proposed in [73]. The unlabeled data was first automatically labeled using labeled data. Newly labeled data and initial labeled data were used to train the deep CNN. The method used semi-supervised deep CNN for breast cancer diagnosis. The performance of CNN was compared with SVM and ANN using different numbers of labeled data like 40, 70 and 100. The model produced comparable results even with sparse labeled data with accuracy of $83 \%$ and AUC of $88 \%$.

\subsection{Heart diseases}

\subsubsection{Pyramid of scales localization}

Pyramid of scales (PoS) localization leads to better performance, specially in cases, where the size of the organ varies between the patients. The size of the heart is not consistent among human, and hence PoS was proposed for localization of left ventricle (LV) in cardiac MRI images in [17].
The model also exploited patch based training. Evaluation metrics used were accuracy, sensitivity and specificity and were respectively $98.6 \%, 83.9 \%$ and $99.1 \%$. The limitation of the approach was the computing time of $10 \mathrm{~s} / \mathrm{image}$.

\subsection{Fetal abnormalities}

\subsubsection{Transfer learning CNN}

Transfer learning uses the knowledge of low layers of a base CNN trained on a large cross domain of dataset of images. Transfer learning advantages include saving of training time, and need of less data for training. This reduces overfitting and enhances the classification performance. Domain transferred deep CNN for fetal abdominal standard plane (FASP) localization in fetal ultrasound scanning was proposed in [10]. The base CNN was trained on 2014 ImageNet detection dataset. The metrics accuracy, precision, recall and F-score were the highest when compared to R-CNN and RVD. The drawback of the system was that it took more time to locate FASP from one ultra sound video. The CNN methods for image localization previewed in this paper are summarized in Table 14.

The papers reviewed for medical image understanding are summarized in Table 15.

\section{Critical review and conclusion}

CNNs have been successfully applied in the areas of medical image understanding and this section provides a critical review of applications of CNNs in medical image understanding. Firstly, the literature contains a vast number of $\mathrm{CNN}$ architectures. It is difficult to select the best architecture for a specific task due to high diversity in architectures.

Table 13 A summary of CNN applications in medical image detection surveyed in Sect. 6

\begin{tabular}{|c|c|c|c|c|}
\hline References & Organ & Preprocessing & Architecture & Performance metric \\
\hline [76] & Breast & $\begin{array}{l}\text { Threshold tissue from white } \\
\text { background and patch-based clas- } \\
\text { sification }\end{array}$ & GoogLeNet & $\begin{array}{l}\text { AUC for slide based classification } \\
92.5 \% \text {, lesion based detection } \\
\text { sensitivity } 70.5 \%\end{array}$ \\
\hline [50] & Skin (melanoma) & $\begin{array}{l}\text { Illumination correction step, } \\
\text { removal of noise using gaussian } \\
\text { filters }\end{array}$ & $\begin{array}{l}\text { (Conv, ReLU, maxpool) } \times 2, F C, \\
\text { softmax }\end{array}$ & $\begin{array}{l}\text { Sensitivity, specificity, true positive, } \\
\text { false positive and accuracy }\end{array}$ \\
\hline [75] & Hemorrhage (eye) & $\begin{array}{l}\text { Image contrast using gaussian } \\
\text { filters, augment using vertical, } \\
\text { horizontal flips }\end{array}$ & $\begin{array}{l}\text { (Conv, ReLU, maxpool) } \times 5, F C, \\
\text { softmax }\end{array}$ & Sensitivity, specificity and ROC \\
\hline [46] & Eye retina & & $\begin{array}{l}\text { Ensemble of twelve } \mathrm{CNN} \text { having } \\
(\text { conv, ReLU) } \times 3 \text {, (FC, dropout }) \\
\times 2\end{array}$ & Accuracy, FROC of 0.928 \\
\hline [69] & Cell & $\begin{array}{l}\text { Extraction of grayscale patches, } \\
\text { segmentation and binarization }\end{array}$ & LeNet & $\begin{array}{l}\text { Sensitivity, specificity, AUC and } \\
\text { F-score. F-score (SVM 78\%, CNN } \\
89 \% \text { ) }\end{array}$ \\
\hline
\end{tabular}


Table 14 A summary of CNN applications in medical image localization surveyed in Sect. 7

\begin{tabular}{|c|c|c|c|c|c|}
\hline References & Organ & Architecture & Dataset & Comparison against & Performance Metric \\
\hline [17] & Heart & $\begin{array}{l}(\text { Conv, ReLU, maxpool }) \times 2 \text {, } \\
\text { FC }\end{array}$ & $\begin{array}{l}\text { Public dataset of York } \\
\text { University }\end{array}$ & & $\begin{array}{r}\text { Accuracy } 98.66 \% \text {, sensitivity } \\
83.91 \% \text {, specificity } 99.07 \%\end{array}$ \\
\hline [10] & Fetal abdominal & $\begin{array}{r}(\text { Conv, ReLU, maxpool }) \times 2 \\
\text { conv } \times 3, \text { maxpool, FC } \times 3\end{array}$ & $\begin{array}{l}\text { Imagenet 2014, Shenzen } \\
\text { Maternal and Child } \\
\text { Healthcare Hospital, China }\end{array}$ & R-CNN and RVD & $\begin{array}{l}\text { Accuracy, precision, recall, } \\
\text { F-score }\end{array}$ \\
\hline [73] & Breast & Semi-supervised deep CNN & & SVM, ANN & Accuracy $83 \%$, AUC $88 \%$ \\
\hline
\end{tabular}

Table 15 Summary of papers reviewed for CNN applications in medical image understanding

\begin{tabular}{|c|c|c|}
\hline Image understanding tasks & Organs targeted & References \\
\hline \multirow[t]{9}{*}{ Medical image classification } & Lung & {$[4,18,44,68]$} \\
\hline & COVID-19 & {$[6,13,22,24,45]$} \\
\hline & HEp-2 & {$[7,21]$} \\
\hline & Breast & {$[34,52,73,78]$} \\
\hline & Heart & {$[20,40]$} \\
\hline & Eye & [57] \\
\hline & Digestive organs & [82] \\
\hline & Brain & {$[19,63]$} \\
\hline & Colon & {$[61,71,78]$} \\
\hline \multirow[t]{6}{*}{ Medical image segmentation } & Brain & {$[25,37,49,55,66]$} \\
\hline & Breast & {$[36,72]$} \\
\hline & MS lesion & [8] \\
\hline & Nucleus & [77] \\
\hline & Heart & {$[83]$} \\
\hline & Eye & {$[81]$} \\
\hline \multirow[t]{4}{*}{ Medical image detection } & Breast & {$[5,76]$} \\
\hline & Melanoma & {$[50]$} \\
\hline & Eye & {$[46,75]$} \\
\hline & Cell & [69] \\
\hline \multirow[t]{2}{*}{ Medical Image localization } & Heart & {$[17]$} \\
\hline & Fetal Abdomen & [10] \\
\hline
\end{tabular}

Moreover, the same architecture might result in different performance due to inefficient data preprocessing techniques. A prior knowledge of data for applying the correct preprocessing technique is needed. Futhermore, hyper parameters optimization (dropout rate, learning rate, optimizer etc) help in enhancing or declining the performance of a network.

For training, CNNs require exhaustive amounts of data containing the most comprehensive information. Insufficient information or features leads to underfitting of the model. However, augmentation could be applied in such scenarios as it results in translation variance and increases the training dataset, thereby enhancing the CNNs efficiency. Furthermore, transfer learning and fine-tuning could also be used to enhance the efficiency in case of sparse availability of data. These enhance the performance since the low level features are nearly the same for most of the images.
Small-sized kernels could be used to enhance the performance by capturing low-level textual information. However, it is at the cost of increased computational complexity during training. Moreover, multiple pathway architecture could be used to enhance performance of CNN. The performance is enhanced due to simultaneous learning of global contextual features and local detailed features, but this in turn, increases the computational burden on the processor and memory.

One of the challenge involved in medical data is the class imbalance problem, where the positive class is generally under-represented and most of the images belong to the normal class. Designing CNNs to work on imbalanced data is a challenging task. However, researchers have tried to overcome this challenge by applying augmentation of the under-represented data. Denser CNNs could also lead to the vanishing gradient problem which could be overcome by using skip connections as in the inceptionNet architecture.

Furthermore, CNNs' significant depth and enormous size require huge memory and higher computational resources for training. The deeper CNNs involves millions of training parameters which could lead the model to overfit and also inefficient at generalization, especially in the case of limited dataset. This calls for models which are lightweight and which could also extract critical features like the dense models. Lightweight CNNs could be explored further.

Medical image Understanding would be more efficient in the presence of background context or knowledge about the image to be understood. In this context, CNNs would be more efficient if the data consists of not only images, but also patient history. Hence, the next challenging task would be to build models, which take as input both images and patient history to make a decision and this could be the next research trend.

Interpreting $\mathrm{CNNs}$ is challenging due to many layers, millions of parameters, and complex, nonlinear data structures. CNN researchers have been concentrating on building accurate models without quantifying uncertainty in the obtained results. The need for successful utilization of the CNN model in medical diagnosis lies in providing confidence and this confidence needs the ability of the model to ascertain its uncertainty or certainty or explain the results obtained. This field needs further exploration. Although, researchers have proposed heat maps, class activation maps 
Table 16 Ways of addressing challenges of medical image understanding

\begin{tabular}{ll}
\hline Challenges in medical image understanding & Solutions for overcoming challenges \\
\hline Less training samples & Augmentation \\
Sparsity of labeled data & Semi supervised deep CNN \\
Noise in images & Gaussian filtering \\
Thicker CT images of brain & Geometric normalization \\
Extract local and global features & Multipathway CNN \\
Avoid redundant computations & ROI segmentation using fCNN \\
Reduce classification errors & Train on misclassified image patches \\
Heart classification & Two path CNN, for spatial and temporal features \\
Imbalanced label distributions & Two phase training \\
Less resolution in brain images & Slice by slice segmentation \\
Increase accuracy and AUC in eye images (retinal eye & Ensemble learning (boosting instead of back \\
vessel) & propagation, RF instead of FC) \\
\hline
\end{tabular}

(CAM), grad CAM, grad CAM++ for visualization of $\mathrm{CNN}$ outputs, the area of visualization is still a challenge.

The various challenges and methods of overcoming some of the challenges of medical image understanding are summarized in Table 16. Further, efficient architectures to overcome some of the challenges as per the survey are summarized in Table 17.
Deep learning includes methods like $\mathrm{CNN}$, recurrent neural network and generative adversial networks. The review of these methods and applications have not been included, as these methods by themselves become topics of research and there is lot of research happening in those areas. Moreover, all the aspects involved in medical image understanding are

Table 17 Efficient CNN architectures For medical image understanding

\begin{tabular}{|c|c|c|c|}
\hline Organ (modality) & Diseases & Tasks & Best architecture \\
\hline \multirow[t]{4}{*}{ Brain MRI } & \multirow[t]{2}{*}{ Alzheimer } & Segementation & Lenet-5, GoogleNet \\
\hline & & Detection & Multipath CNN \\
\hline & Epilepsy & Classification & $\mathrm{CNN}$ with seven conv and \\
\hline & Schizophrenic, bipolar disorder & Segementation & Patch based CNN with 4 conv, 2 maxpool and softmax regression \\
\hline \multirow[t]{4}{*}{ Breast mammogram } & \multirow[t]{4}{*}{ Tumour } & Segementation & $($ Conv + maxpool $) \times 2+2$ FC, Softmax \\
\hline & & Detection & Scaled VGGnet or GoogleNet \\
\hline & & Classification & Alexnet \\
\hline & & Localization & Semi Supervised Deep CNN \\
\hline Heart (CT) & Coronary artery calcium scoring & Segmentation & Two path CNN \\
\hline Heart (ECG) & ECG & Classification & $\begin{array}{l}\text { Two path CNN, one along spatial and the other along temporal } \\
\text { and fused together finally }\end{array}$ \\
\hline Heart (MRI) left ventricle & & Localization & $($ Conv, maxpool $) \times 2, \mathrm{FC}$ \\
\hline \multirow[t]{4}{*}{ Lung (CT) } & Cancer & Classification & Ensemble of overfeat across axial, saggital and coronal plane \\
\hline & Nodule & Detection & Lenet, Overfeat \\
\hline & COVID-19 & Classification & multi scale multi encoder ensemble CNN model \\
\hline & ILD & Classification & Any $\mathrm{CNN}$ architecture like Alexnet \\
\hline Hep-2 cell & & Classification & (Conv, maxpool) $\times 3,2 \mathrm{FC}$, softmax regressor \\
\hline \multirow[t]{3}{*}{ Eye } & Haemorrhage & Detection & (Conv, ReLu, maxpool) $\times 5$, FC, softmax classifier \\
\hline & Glaucoma & Detection & $($ Conv, ReLu, maxpool) $\times 2$, FC with adaboost \\
\hline & Retinopathy & Segmentation & (Conv, ReLu, maxpool) $\times 10,3$ FC, softmax classifier \\
\hline \multirow[t]{2}{*}{ Colon } & Polyp & Classification & Any simple $\mathrm{CNN}$ architecture \\
\hline & Polyp & Detection & Ensemble of CNN \\
\hline Skin & Melanoma & Detection & $($ Conv, ReLu, maxpool) $\times 2, \mathrm{FC}$ \\
\hline Liver $(\mathrm{CT})$ & & Classification & (Conv, maxpool) $\times 2,3$ conv, max pool, 3 FC, softmax \\
\hline Abdomen (US scan) & Fetus & Localization & $($ Conv, ReLU, maxpool $) \times 2$ conv $\times 3$, maxpool, $\mathrm{FC} \times 3$ \\
\hline
\end{tabular}


Fig. 2 Bar chart summarizing the number of papers surveyed

\section{Summary of papers}

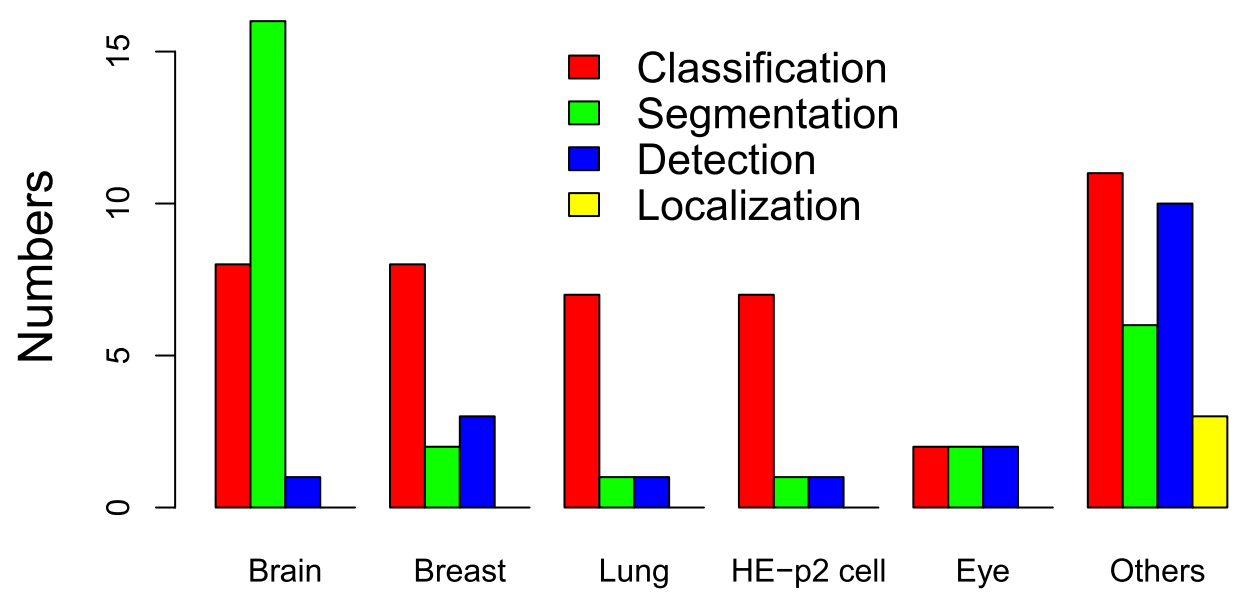

also not included since it is an ocean and the focus in the paper is only on a few important techniques involved.

\section{Conclusion}

The heterogeneous nature of medical anomalies in terms of shape, size, appearance, location and symptoms poses challenges for medical anomaly diagnosis and prognosis. Traditional methods of using human specialists involve fatigue, oversight and high cost and also sparse availability. ML-based healthcare systems need efficient feature extraction methods. But efficient features are still unknown and also, the methods available for feature extraction are not very efficient. This calls for intelligent healthcare systems that automatically extract efficient features for medical image understanding that aids diagnosis and prognosis. $\mathrm{CNN}$ is a popular technique for solving medical image understanding challenges due to its highly efficient methods of feature extraction and learning low level, mid level and high level discriminant features of an input medical image.

The literature reviewed in this paper underscores that researchers have focused their attention on the use of CNN to overcome many challenges in medical image understanding. Many have accomplished the task successfully. The CNN methods discussed in this paper have been found to either outperform or compliment the existing traditional and ML approaches in terms of accuracy, sensitivity, AUC, DSC, time taken etc. However, their performance is often not the best due to a few factors. A snapshot summary of the quantum of research articles surveyed in this article is presented in the Fig. 2.

The challenges in image understanding with respect to medical imaging have been discussed in this paper. Various image understanding tasks have been introduced. In addition, $\mathrm{CNN}$ and its various components have been outlined briefly. The approaches used by the researchers to address the various challenges in medical image understanding have been surveyed.

$\mathrm{CNN}$ models have been described as black boxes and there is a lot of research happening in terms of analyzing and understanding output at every layer. Since medical images are involved, we need an accountable and efficient prediction system which should also be able to articulate about a decision taken. Researchers are also working on image captioning (textual representations of the image) [29]. This will enable physicians to understand the perception of the network at both output layer and intermediate levels. Researchers have tried Bayesian deep learning models which calculates the uncertainty estimates [38]. This would help physicians assess the model. All these could further accelerate medical image understanding using CNNs among physicians.

Acknowledgements The authors acknowledge with gratitude the support received from REVA University, Bengaluru, and M. S. Ramaiah University of Applied Sciences, Bengaluru, India.

\section{Compliance with ethical standards}

Conflict of interest The authors declare that they have no conflict of interest.

\section{References}

1. Abadi M, Agarwal A, Barham P (2016) Tensorflow: large-scale machine learning on heterogeneous distributed systems. CoRR Arxiv: 1603.04467

2. Akkus Z, Galimzianova A, Hoogi A, Rubin DL, Erickson BJ (2017) Deep learning for brain MRI segmentation: state of the art and future directions. J Digit Imaging 30(4):449-459

3. Al-Rfou R, Alain G, Almahairi A, Angermueller C, Bahdanau D, Ballas N, Bengio Y (2016) Theano: a python framework for fastcomputation of mathematical expressions. arXiv: 1605.02688 
4. Anthimopoulos M, Christodoulidis S, Ebner L, Christe A, Mougiakakou SG (2016) Lung pattern classification for interstitial lung diseases using a deep convolutional neural network. IEEE Trans Med Imaging 35(5):1207-1216

5. Apou G, Schaadt NS, Naegel B (2016) Structures in normal breast tissue. Comput Biol Med 74:91-102

6. Bassi PAS, Attux R (2020) A deep convolutional neural network for COVID-19 detection using chest X-rays

7. Bayramoglu N, Kannala J, Heikkilä J (2015) Human epithelial type 2 cell classification with convolutional neural networks. In: Proceedings of the 15th IEEE international conference on bioinformatics and bioengineering, BIBE, pp 1-6

8. Brosch T, Tang LYW, Yoo Y, Li DKB, Traboulsee A, Tam RC (2016) Deep 3-D convolutional encoder networks with shortcuts for multiscale feature integration applied to multiple sclerosis lesion segmentation. IEEE Trans Med Imaging 35(5):1229-1239

9. CS231N convolutional neural network for visual recogntion (2019). http://cs231n.stanford.edu/. Accessed 24 June 2019

10. Chen H, Ni D, Qin J (2015) Standard plane localization in fetal ultrasound via domain transferred deep neural networks. IEEE J Biomed Health Inform 19(5):1627-1636

11. Chollet F (2018) Keras: the Python deep learning library. https:// keras.io/. Accessed 24 June 2019

12. Chollet F (2017) Xception: deep learning with depthwise separable convolutions. In: Proceedings of the IEEE conference on computer vision and pattern recognition, (CVPR), pp 1800-1807

13. Chowdhury NK, Rahman MM, Kabir MA (2020) Pdcovidnet: a parallel-dilated convolutional neural network architecture for detecting COVID-19 from chest X-ray images

14. Ciompi F, de Hoop B, van Riel SJ (2015) Automatic classification of pulmonary peri-fissural nodules in computed tomography using an ensemble of 2-D views and a convolutional neural network out-of-the-box. Med Image Anal 26(1):195-202. https://doi. org/10.1016/j.media.2015.08.001

15. Collobert R, Kavukcuoglu K, Farabet C (2011) Torch7: a Matlablike environment for machine learning. In: BigLearn, NIPS workshop, EPFL-CONF-192376

16. Dasgupta A, Singh S (2017) A fully convolutional neural network based structured prediction approach towards the retinal vessel segmentation. In: Proceedings of the 14th IEEE international symposium on biomedical imaging (ISBI), pp 248-251

17. Emad O, Yassine IA, Fahmy AS (2015) Automatic localization of the left ventricle in cardiac MRI images using deep learning. In: Proceedings of the 37th IEEE annual international conference on engineering in medicine and biology society (EMBC), pp 683-686

18. Gao M, Bagci U, Lu L, Wu A, Buty M (2018) Holistic classification of CT attenuation patterns for interstitial lung diseases via deep convolutional neural networks. CMBBE Imaging Vis 6(1): $1-6$

19. Gao XW, Hui R (2016)A deep learning based approach to classification of CT brain images. In: Proceedings of the SAI computing conference, pp 28-31

20. Gao XW, Li W, Loomes M, Wang L (2017) A fused deep learning architecture for viewpoint classification of echocardiography. Inf Fusion 36:103-113

21. Gao Z, Zhang J, Zhou L, Wang L (2014) HEp-2i cell image classification with deep convolutional neural networks. IEEE J Biomed Health Inform 21:416-428

22. Ghoshal B, Tucker A (2020) Estimating uncertainty and interpretability in deep learning for coronavirus (COVID-19) detection arXiv:2003.10769

23. Gordienko Y, Gang P, Hui J, Zeng W, Kochura Y, Alienin O, Rokovyi O, Stirenko S (2017) Deep learning with lung segmentation and bone shadow exclusion techniques for chest X-ray analysis of lung cancer. CoRR arXiv: 1712.07632
24. Hasan MK, Alam MA, Elahi MTE, Roy S, Wahid SR (2020) CVR-NET: a deep convolutional neural network for coronavirus recognition from chest radiography images

25. Havaei M, Davy A, Warde-Farley D (2017) Brain tumor segmentation with deep neural networks. Med Image Anal $35: 18-31$

26. He K, Zhang X, Ren S, Sun J (2016) Deep residual learning for image recognition. In: Proceedings of the IEEE conference on computer vision and pattern recognition, (CVPR), pp 770-778

27. Hesamian M, Jia W, He X (2019) Deep learning techniques for medical image segmentation: achievements and challenges. J Digit Imaging 32:582-596

28. Ho TK (1995) Random decision forests. In: Proceedings of the 3rd IEEE international conference on document analysis and recognition, vol 1, pp 278-282

29. Hossain MZ, Sohel F, Shiratuddin MF, Laga H (2018) A comprehensive survey of deep learning for image captioning. CoRR arXiv: 1810.04020

30. Hubel D, Wiesel T (1959) Receptive fields of single neurones in the cat's striate cortex. J Physiol 148(3):574-591

31. ICPR 2018 international conference on pattern recognition (2019). http://www.icpr2018.org. Accessed 24 June 2019

32. ISLES challenge 2018 ischemic stroke lesion segmentation (2018). http://www.isles-challenge.org. Accessed 24 June 2019

33. Jia, Y., Shelhamer E, Donahue J (2014) Caffe: convolutional architecture for fast feature embedding. In: Proceedings of the 22th ACM international conference on multimedia, MM '14, pp 675-678. ACM, New York

34. Jiao Z, Gao X, Wang Y, Li J (2016) A deep feature based framework for breast masses classification. Neurocomputing 197:221-231

35. Jusman Y, Ng SC, Abu Osman NA (2014) Intelligent screening systems for cervical cancer. Sci World J 2014:810368

36. Kallenberg M, Petersen K, Nielsen M (2016) Unsupervised deep learning applied to breast density segmentation and mammographic risk scoring. IEEE Trans Med Imaging 35(5):1322-1331

37. Kamnitsas K, Ferrante E, Parisot S (2016) Deepmedic for brain tumor segmentation. In: Proceedings of the international workshop on brainlesion: glioma, multiple sclerosis, stroke and traumatic brain injuries. Springer, pp 138-149

38. Kendall A, Gal Y (2017) What uncertainties do we need in bayesian deep learning for computer vision? CoRR arXiv:1703.04977

39. Kermany DS, Goldbaum M, Cai W, Valentim CC, Liang H, Baxter SL, McKeown A, Yang G, Wu X, Yan F et al (2018) Identifying medical diagnoses and treatable diseases by image-based deep learning. Cell 172(5):1122-1131

40. Kiranyaz S, Ince T, Gabbouj M (2016) Real-time patient-specific ECG classification by 1-D convolutional neural networks. IEEE Trans Biomed Eng 63(3):664-675

41. Krizhevsky A, Sutskever I, Hinton GE (2012) Imagenet classification with deep convolutional neural networks. In: Advances in neural information processing systems 25 : proceedings of the 26th annual conference on neural information processing systems, pp $1106-1114$

42. Larochelle H, Bengio Y (2008) Classification using discriminative restricted Boltzmann machines. In: Proceedings of the 25th international conference on machine learning, pp 536-543

43. LeCun Y, Bottou L, Bengio Y, Haffner P (1998) Gradientbased learning applied to document recognition. Proc IEEE 86(11):2278-2324

44. Li Q, Cai W, Wang X, Zhou Y, Feng DD, Chen M (2014) Medical image classification with convolutional neural network. In: Proceedings of the 13th international conference on control, automation, robotics and vision (ICARCV), pp 844-848

45. Maghdid HS, Asaad AT, Ghafoor KZ, Sadiq AS, Khan MK (2020) Diagnosing COVID-19 pneumonia from X-ray and CT images 
using deep learning and transfer learning algorithms. CoRR arXiv :2004.00038

46. Maji D, Santara A, Mitra P (2016) Ensemble of deep convolutional neural networks for learning to detect retinal vessels in fundus images. arXiv preprint arXiv:1603.04833

47. Mavroforakis ME, Georgiou HV, Dimitropoulos N (2006) Mammographic masses characterization based on localized texture and dataset fractal analysis using linear, neural and support vector machine classifiers. Artif Intell Med 37(2):145-162

48. Menze BH, Jakab A, Bauer S (2015) The multimodal brain tumor image segmentation benchmark (BRATS). IEEE Trans Med Imaging 34(10): 1993-2024

49. Moeskops P, Viergever MA, Mendrik AM, de Vries LS, Benders MJNL, Isgum I (2016) Automatic segmentation of MR brain images with a convolutional neural network. IEEE Trans Med Imaging 35(5):1252-1261

50. Nasr-Esfahani E, Samavi S, Karimi N (2016) Melanoma detection by analysis of clinical images using convolutional neural network. In: Proceedings of the 38th IEEE annual international conference on engineering in medicine and biology society, EMBC, pp 1373-1376

51. Nervana Systems Inc (2018) neon. http://neon.nervanasys.com/ docs/latest/. Accessed 24 June 2019

52. Ovalle JEA, González FA, Ramos-Pollán R, Oliveira JL, GuevaraLópez MÁ (2016) Representation learning for mammography mass lesion classification with convolutional neural networks. Comput Methods Prog Biomed 127:248-257

53. Paszke A, Gross S, Chintala S, Chanan G (2018) Tensors and dynamic neural networks in Python with strong GPU acceleration. https://pytorch.org/. Accessed 24 June 2019

54. Pereira S, Pinto A, Alves V, Silva CA (2015) Deep convolutional neural networks for the segmentation of gliomas in multi-sequence MRI. In: Brainlesion: glioma, multiple sclerosis, stroke and traumatic brain injuries-first international workshop, Brainles 2015, held in conjunction with MICCAI 2015, Munich, Germany, October 5, 2015, revised selected papers, pp 131-143

55. Pereira S, Pinto A, Alves V, Silva CA (2016) Brain tumor segmentation using convolutional neural networks in MRI images. IEEE Trans Med Imaging 35(5):1240-1251

56. Phillips NA, Rajpurkar P, Sabini, M, Krishnan R, Zhou, S, Pareek, A, Phu NM, Wang C, Ng AY, Lungren MP (2020) Chexphoto: $10,000+$ smartphone photos and synthetic photographic transformations of chest X-rays for benchmarking deep learning robustness. CoRR arXiv:2007.06199

57. Pratt H, Coenen F, Broadbent DM, Harding SP, Zheng Y (2016) Convolutional neural networks for diabetic retinopathy. In: Proceedings of the 20th conference on medical image understanding and analysis, MIUA, pp 200-205

58. Rajpurkar P, Irvin J, Zhu K, Yang B, Mehta H, Duan T, Ding DY, Bagul A, Langlotz C, Shpanskaya KS, Lungren MP, Ng AY (2017) Chexnet: radiologist-level pneumonia detection on chest X-rays with deep learning. CoRR arXiv:1711.05225

59. Ranzato M, Hinton GE, LeCun Y (2015) Guest editorial: deep learning. Int J Comput Vis 113(1):1-2

60. Ravi D, Wong C, Deligianni F, Berthelot M, Pérez JA, Lo B, Yang $G$ (2017) Deep learning for health informatics. IEEE J Biomed Health Inform 21(1):4-21

61. Ribeiro E, Uhl A, Häfner M (2016) Colonic polyp classification with convolutional neural networks. In: Proceedings of the 29th IEEE international symposium on computer-based medical systems, (CBMS), pp 253-258

62. Sajjad M, Khan S, Muhammad K, Wu W, Ullah A, Baik SW (2019) Multi-grade brain tumor classification using deep CNN with extensive data augmentation. J Comput Sci 30:174-182. https://doi.org/10.1016/j.jocs.2018.12.003
63. Sarraf S, Tofighi G (2016) Classification of Alzheimer's disease using fMRI data and deep learning convolutional neural networks. Computer Research Repository. arXiv:1603.08631

64. Seide F, Agarwal A (2016) CNTK: Microsoft's open-source deeplearning toolkit. In: Proceedings of the 22nd ACM international conference on knowledge discovery and data mining, p 2135

65. Sermanet P, Eigen D, Zhang X, Mathieu M, Fergus R, LeCun Y (2013) Overfeat: integrated recognition, localization and detection using convolutional networks. Computing Research Repository. arXiv: 1312.6229

66. Shakeri M, Tsogkas S, Ferrante E, Lippe S, Kadoury S, Paragios N, Kokkinos I (2016) Sub-cortical brain structure segmentation using F-CNNs. In: Proceedigns of the IEEE 13th international symposium on biomedical imaging (ISBI). IEEE, pp 269-272

67. Shankar K, Zhang Y, Liu Y, Wu L, Chen CH (2020) Hyperparameter tuning deep learning for diabetic retinopathy fundus image classification. IEEE Access 8:118164-118173

68. Shen W, Zhou M (2017) Multi-crop convolutional neural networks for lung nodule malignancy suspiciousness classification. Pattern Recognit 61:663-673

69. Shkolyar A, Gefen A, Benayahu D, Greenspan H (2015) Automatic detection of cell divisions (mitosis) in live-imaging microscopy images using convolutional neural networks. In: Proceedings of the 37th annual international conference of the IEEE engineering in medicine and biology society (EMBC), pp 743-746

70. Simonyan K, Zisserman A (2014) Very deep convolutional networks for large-scale image recognition. Computer Research Repository. arXiv: 1409.1556

71. Sirinukunwattana K, Raza SEA, Tsang Y, Snead DRJ, Cree IA, Rajpoot NM (2016) Locality sensitive deep learning for detection and classification of nuclei in routine colon cancer histology images. IEEE Trans Med Imaging 35(5):1196-1206

72. Su H, Liu F, Xie Y (2015) Region segmentation in histopathological breast cancer images using deep convolutional neural network. In: Proceedings of the 12th IEEE international symposium on biomedical imaging (ISBI), pp 55-58

73. Sun W, Tseng TB, Zhang J, Qian W (2017) Enhancing deep convolutional neural network scheme for breast cancer diagnosis with unlabeled data. Comput Med Imaging Graph 57:4-9

74. Szegedy C, Liu W, Jia Y, Sermanet P, Reed SE (2015) Going deeper with convolutions. In: Proceedings of the IEEE conference on computer vision and pattern recognition (CVPR), pp 1-9

75. van Grinsven MJ, van Ginneken B, Hoyng CB (2016) Fast convolutional neural network training using selective data sampling: application to hemorrhage detection in color fundus images. IEEE Trans Med Imaging 35(5):1273-1284

76. Wang D, Khosla A, Gargeya R, Irshad H, Beck AH (2016) Deep learning for identifying metastatic breast cancer. Computer Research Repository. arXiv: 1606.05718

77. Xing F, Xie Y, Yang L (2016) An automatic learning-based framework for robust nucleus segmentation. IEEE Trans Med Imaging 35(2):550-566

78. Xu J, Luo X, Wang G, Gilmore H, Madabhushi A (2016) A deep convolutional neural network for segmenting and classifying epithelial and stromal regions in histopathological images. Neurocomputing 191:214-223

79. Zeiler MD, Fergus R (2013) Visualizing and understanding convolutional networks. Computer Research Repository. arXiv $: 1311.2901$

80. Zhao L, Jia K (2016) Multiscale CNNs for brain tumor segmentation and diagnosis. Comput Math Methods Med 2016:8356294:1-8356294:7

81. Zilly JG, Buhmann JM, Mahapatra D (2017) Glaucoma detection using entropy sampling and ensemble learning for automatic optic cup and disc segmentation. Comput Med Imaging Graph $55: 28-41$ 
82. Zou Y, Li L, Wang Y (2015) Classifying digestive organs in wireless capsule endoscopy images based on deep convolutional neural network. In: Proceedings of the IEEE international conference on digital signal processing, DSP, pp 1274-1278

83. Zreik M, Leiner T, de Vos BD, van Hamersvelt RW, Viergever MA, Isgum I (2016) Automatic segmentation of the left ventricle in cardiac CT angiography using convolutional neural networks.
In: Proceedings of the 13th IEEE international symposium on biomedical imaging (ISBI), pp 40-43

Publisher's Note Springer Nature remains neutral with regard to jurisdictional claims in published maps and institutional affiliations. 